\title{
Kinematics and Force Analysis of Lifting Mechanism of Detachable Container Garbage Truck
}

\author{
Shanzeng Liu* and Lianjie Zhang
}

\author{
School of Mechatronic Engineering, China University of Mining and Technology, Xuzhou 221116, P.R. China
}

\begin{abstract}
The kinematics and force analysis of the lifting mechanism of the detachable container garbage truck is performed in this article. First of all, the structural features and working mechanism of detachable container garbage truck are introduced. Then, for each working condition of the DCG truck, kinematic models are established, and the force analysis of the lifting mechanism is performed. Finally, the movement of a 20 ton DCG truck is calculated and analyzed as an example. This study provides a theoretical basis for the optimized design of DCGgarbage truck.
\end{abstract}

Keywords: Detachable container garbage truck, lifting mechanism, kinematic analysis, force analysis.

\section{INTRODUCTION}

With the rapid economic and social development in China, the amount of garbage increases dramatically every year. Many large cities are besieged by garbage. How to quickly and efficiently treat the municipal solid waste becomes a major problem confronted by us. DCG truck is a high-efficiency waste transfer vehicle which consists of automobile chassis, lifting mechanism and a garbage container $[1,2]$. The lifting mechanism (namely, link mechanism) performs the functions of automatic loading and unloading of the container and garbage dumping. It is now widely applied in garbage treatment as well as in the loading, transport and unloading of a variety of commodities $[3,4]$. At present, the commercially available DCG trucks on China's market are small tonnage vehicles ( 3 ton or 5 ton). The trucks are usually designed by surveying and drawing or empirical value setting, which severely restricts the improvement of the product performance and the promotion of market application $[5,6]$.

The structural features of the DCG truck are analyzed, and its working mechanism is also analyzed. The kinematic and mechanical models are established corresponding to the loading and unloading of the DCG garbage truck. Using a 20 t DCG truck as an example, the kinematic and mechanical models are analyzed and solved.

\section{STRUCTURE AND WORKING MECHANISM}

The DCG truck is divided into three types, a straight arm type, swing arm type (Fig. 1) and a slide type. The structural diagram of the lifting mechanism of the swing arm type is shown in Fig. (2).

In Fig. (2), point $A$ is the hinge joint between the auxiliary frame and lifting cylinder 1 ; point $B$ is the hinge joint between the guide pulley of auxiliary frame 7 and the auxiliary frame; point $C$ is the contact point between the

*Address correspondence to this author at the School of Mechatronic Engineering, China University of Mining and Technology, Xuzhou 221116, P.R. China; Tel: +8615252026399; E-mail: liushanzeng@163.com guide pulley of auxiliary frame 7 and the bottom of garbage container 6; point $D$ is the hinge point between the flip frame 3 and the lifting arm 4; point $E$ is the hinge point between the lifting arm 4 and the lifting cylinder 1 ; point $F$ is the hinge point between the swing arm cylinder 2 and the lifting arm 4; point $G$ is the hinge point between the swing arm 5 and the lifting arm 4; point $H$ is the hinge point between the swing arm cylinder 2 and the swing arm 5; point $M$ is the hinge point between the hook on the swing arm 5 and the hook on the garbage container 5; point $R$ is the contact point between the roller on the bottom of garbage container 6 and the ground 9 .

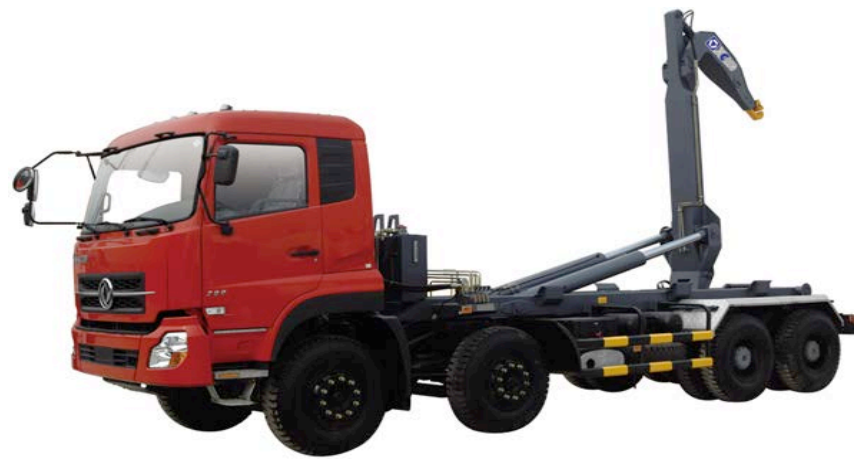

Fig. (1). Detachable container garbage truck.

The DCG truck has four working conditions: loading, transport, garbage dumping and unloading. The principle of container unloading for the swing arm type is described as follows [7]: the swing arm cylinder 2 stretches, then rotates by a certain angle and pushes the garbage container 6 backwards. After the completion of the action of swing arm cylinder the lifting cylinder 1 stretches again to lift the lifting arm 4 and the swing arm 5 so that the bottom of the garbage container comes in contacts with the guide pulley 7 of auxiliary frame and moves backwards. When the roller 8 at the rear of the garbage container gets into contact with ground 9, the bottom of the garbage container is detached from the guide pulley of the auxiliary frame. The rear roller on the garbage container enables the container to roll on the 
ground. In this way, the garbage container is unloaded onto the ground. The working mechanism of container loading is identical with that of container unloading, but in a reverse sequence. To dump the garbage, a locking mechanism locks the lifting arm and flips frame 3 together. When the lifting cylinder stretches, the lifting arm rotates around the hinge point $B$ together with the auxiliary frame. Therefore, the garbage is dumped.

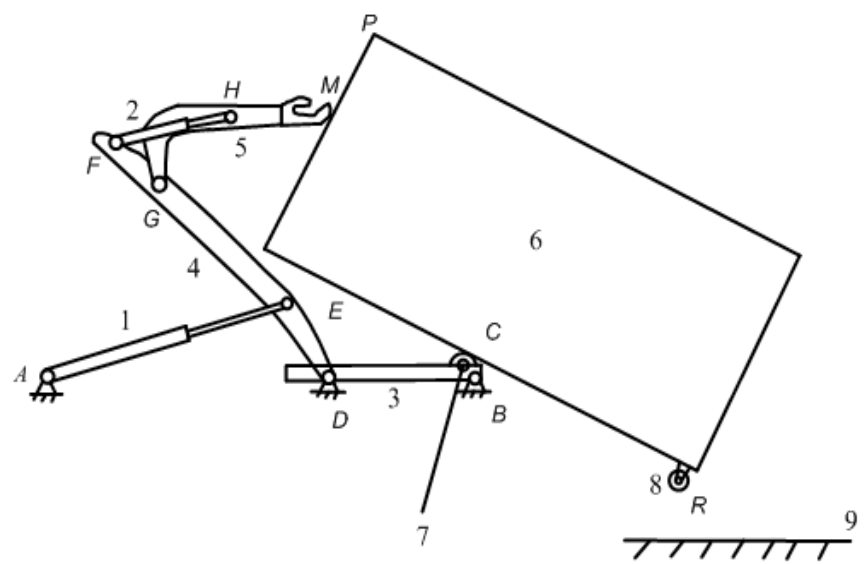

1. Lifting cylinder, 2. Swing arm cylinder, 3. Flip frame, 4. Lifting arm, 5. Swing arm, 6. Garbage container, 7. Guide pulley of flip frame, 8. Container roller, 9. Ground

Fig. (2). Structural diagram of the lifting mechanism.

\section{KINEMATIC AND FORCE ANALYSIS}

To facilitate the analysis of the system of DCG truck, the following simplification and assumptions are made:

(a) Due to the large weight of the fully loaded garbage container, the weight of the lifting mechanism has little influence on the result of force analysis. Therefore, the weight of each component of the lifting mechanism is ignored.

(b) The speed of container loading and unloading is slow, and therefore the influence of the inertia force of the container is ignored.

(c) The hydraulic cylinder is considered to make uniform motion, and the influence of hydraulic power is ignored.

(d) The deformations of chassis, ground and tires under stress are ignored.

The unloading process of an ordinary detachable container garbage truck can be decomposed into two stages. The first stage involves the placing of the garbage container onto the guide pulley of auxiliary frame. The second stage is the detachment of the bottom of container from the guide pulley and the contact of the roller of the container on the ground $[6,7]$.

\subsection{Kinematic Model of the First Stage}

The working condition of the first stage of unloading is shown in Fig. (3). In this stage, the flip frame and the auxiliary frame are tightly combined together via a locking mechanism. The swing arm has flipped by an angle of $\rho^{\circ}$ and is static relative to the lifting arm [6-8]. The lifting cylinder stretches, and the lifting arm rotates around point $D$, thereby pushing the garbage container backwards. At the moment, the bottom of the container is supported by the guide pulley of the auxiliary frame. The container slides backwards along the guide pulley.
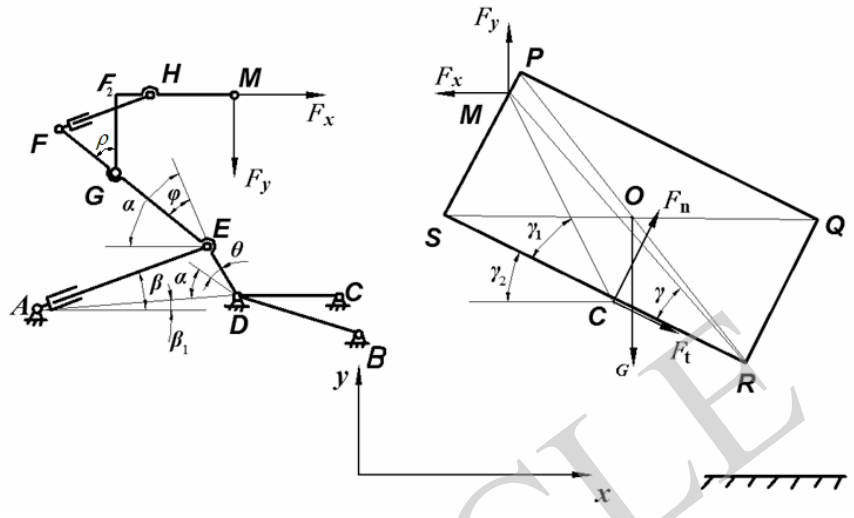

Fig. (3). Schematic diagram of the movement in the first stage.

The direction parallel to the ground is taken as the $X$-axis, and the direction perpendicular to the ground is the $Y$-axis. The rectangular coordinate system is established with the position of point $B$ along $X$-axis as the origin. The garbage container is treated as a standard cuboid, whose geometric center is the center of gravity $O$. The coordinates of each point are shown below:

$$
\begin{aligned}
& \left\{\begin{array}{l}
x_{\mathrm{E}}=x_{\mathrm{D}}-l_{\mathrm{DE}} \cos \left(\alpha+\theta-\beta_{1}\right) \\
y_{\mathrm{E}}=y_{\mathrm{D}}+l_{\mathrm{DE}} \sin \left(\alpha+\theta-\beta_{1}\right)
\end{array}\right. \\
& \left\{\begin{array}{l}
x_{\mathrm{G}}=x_{\mathrm{E}}-l_{\mathrm{EG}} \cos (\alpha-\varphi) \\
y_{\mathrm{G}}=y_{\mathrm{E}}+l_{\mathrm{EG}} \sin (\alpha-\varphi)
\end{array}\right. \\
& \left\{\begin{array}{l}
x_{\mathrm{F}_{2}}=x_{\mathrm{G}}-l_{\mathrm{F}_{2} \mathrm{G}} \cos (\alpha+\rho) \\
y_{\mathrm{F}_{2}}=y_{\mathrm{G}}+l_{\mathrm{F}_{2} \mathrm{G}} \sin (\alpha+\rho)
\end{array}\right. \\
& \left\{\begin{array}{l}
x_{\mathrm{M}}=x_{\mathrm{F}_{2}}+l_{\mathrm{MF}_{2}} \sin (\alpha+\rho) \\
y_{\mathrm{M}}=y_{\mathrm{F}_{2}}+l_{\mathrm{MF}_{2}} \cos (\alpha+\rho)
\end{array}\right.
\end{aligned}
$$


where $\left(x_{\mathrm{D}}, y_{\mathrm{D}}\right),\left(x_{\mathrm{E}}, y_{\mathrm{E}}\right),\left(x_{\mathrm{G}}, y_{\mathrm{G}}\right),\left(x_{\mathrm{F} 2}, y_{\mathrm{F} 2}\right),\left(x_{\mathrm{M}}, y_{\mathrm{M}}\right),\left(x_{\mathrm{R}}, y_{\mathrm{R}}\right)$, $\left(x_{\mathrm{P}}, y_{\mathrm{P}}\right)$ and $\left(x_{\mathrm{O}}, y_{\mathrm{O}}\right)$ are the coordinates of points $D, E, G, F_{2}$, $M, R, P$ and $O$, respectively; $\alpha$ is the angle by which the lifting arms flips in the first stage; $\theta$ and $\varphi$ are the inclusion angles of $D E$ and $E G$ segments on the lifting arm with respect to $X$ axis, respectively, when the lifting arm rotates by zero degree; $\gamma$ is the inclusion angle between $M R$ and $S R$; $\gamma_{1}$ is the inclusion angle between $M C$ and $S C ; \gamma_{2}$ is the inclusion angle between the bottom of garbage container and the $X$-axis; $l_{\mathrm{DE}}$ and $l_{\mathrm{EG}}$ are the lengths of $D E$ and $E G$ segments on the lifting arm, respectively; $l_{\mathrm{MR}}$ and $l_{\mathrm{MP}}$ are the lengths of $M R$ and $M P$, respectively.

The force analysis is made on the lifting mechanism and garbage container of the truck. The force $F_{1}$ of lifting cylinder in the first stage is expressed as follows.

$$
\begin{aligned}
& F_{1}=\left[\frac{\left(\sin \gamma_{2}+\mu_{1} \cos \gamma_{2}\right)\left(y_{\mathrm{M}}-y_{\mathrm{D}}\right)}{\left(y_{\mathrm{E}}-y_{\mathrm{D}}\right) \cos \beta-\left(x_{\mathrm{E}}-x_{\mathrm{D}}\right) \sin \beta}+\right. \\
& \left.\frac{\left(\frac{G}{I_{1}}+\mu_{1} \sin \gamma_{2}-\cos \gamma_{2}\right)\left(x_{\mathrm{M}}-x_{\mathrm{D}}\right)}{\left(y_{\mathrm{E}}-y_{\mathrm{D}}\right) \cos \beta-\left(x_{\mathrm{E}}-x_{\mathrm{D}}\right) \sin \beta}\right] I_{1} \\
& I_{1}=\frac{G\left(x_{\mathrm{O}}-x_{\mathrm{M}}\right)}{\mu_{1} l_{\mathrm{MS}}+\sqrt{\left(x_{\mathrm{M}}-x_{\mathrm{C}}\right)^{2}+\left(y_{\mathrm{M}}-y_{\mathrm{C}}\right)^{2}-l_{\mathrm{MS}}^{2}}}
\end{aligned}
$$

where $\beta$ is the inclusion angle between the lifting cylinder $A E$ and $X$-axis; $\mu_{1}$ is the coefficient of sliding friction between the bottom of garbage container and the guide pulley; $G$ is the total weight of garbage container fully loaded; $l_{\mathrm{MS}}$ is the distance from the suspension point $M$ on the swing arm to the left lower endpoint $S$ of the garbage container; $\gamma_{2}$ is the inclusion angle between the bottom of garbage container and the $X$-axis.

\subsection{Kinematic Model of the Second Stage}

The working condition of the second stage of unloading is shown in Fig. (4). During the second stage, it is ensured that there is no collision and interaction between the guide pulley of the auxiliary frame and the roller $\mathrm{R}$ on the garbage container. This places certain requirements on the parameters of lifting mechanism [6-8].

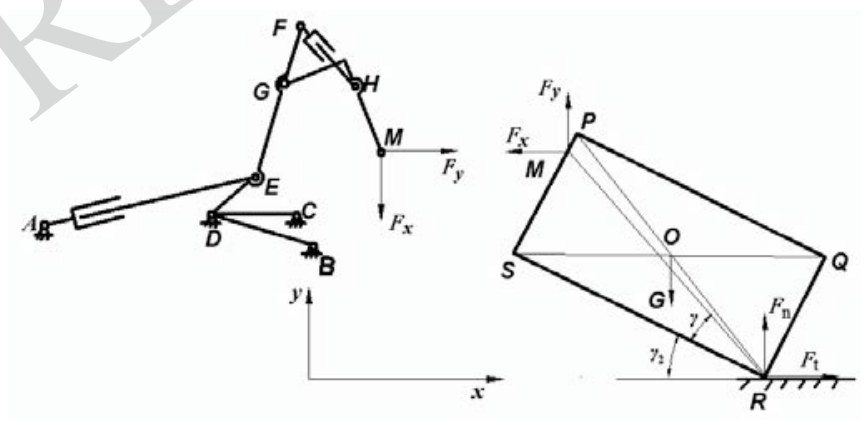

Fig. (4). Schematic diagram of the movement of the second stage

The force analysis is carried out on the lifting mechanism and the garbage container. Thus, the force acting on the lifting cylinder in the second stage is $F_{2}$ :

$$
\begin{aligned}
& F_{2}=\frac{\left[\mu_{2}\left(y_{\mathrm{M}}-y_{\mathrm{D}}\right)+\left(\frac{G}{I_{2}}-1\right)\left(x_{\mathrm{M}}-x_{\mathrm{D}}\right)\right] I_{2}}{\left(y_{\mathrm{E}}-y_{\mathrm{D}}\right) \cos \beta-\left(x_{\mathrm{E}}-x_{\mathrm{D}}\right) \sin \beta} \\
& I_{2}=\frac{G\left(x_{\mathrm{O}}-x_{\mathrm{M}}\right)}{x_{\mathrm{R}}-x_{\mathrm{M}}+\mu_{2}\left(y_{\mathrm{M}}-h_{0}\right)}
\end{aligned}
$$

where, $\mu_{2}$ is the coefficient of sliding friction between the roller on the right lower end of the garbage container and the ground; $h_{0}$ is the vertical distance from the roller of the garbage container to the ground in container unloading [6-8].

\subsection{Kinematic Model of Garbage Dumping}

The working condition of garbage dumping is shown in Fig. (5). At the moment, the lifting arm and the flip frame are combined together and rotate around point $B$.

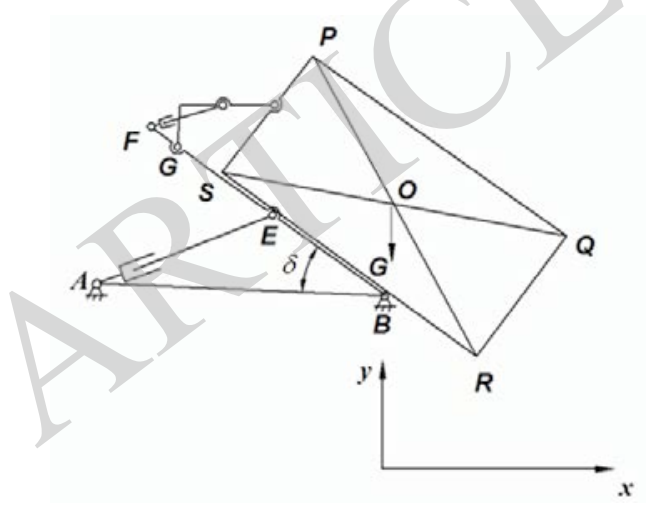

Fig. (5). Mathematical model of garbage dumping.

The thrust exerted by the lifting cylinder is $F_{3}$ when the garbage is dumped. From the force analysis, it can be obtained that

$$
\begin{aligned}
& F_{3}=\frac{G\left(x_{\mathrm{B}}-x_{\mathrm{O}}\right)}{I_{3}} \\
& I_{3}=\frac{\left|\left(y_{\mathrm{E}}-y_{\mathrm{A}}\right) x_{\mathrm{B}}+\left(x_{\mathrm{A}}-x_{\mathrm{E}}\right) y_{\mathrm{B}}+x_{\mathrm{E}} y_{\mathrm{A}}-x_{\mathrm{A}} y_{\mathrm{E}}\right|}{\sqrt{\left(y_{\mathrm{E}}-y_{\mathrm{A}}\right)^{2}+\left(x_{\mathrm{E}}-x_{\mathrm{A}}\right)^{2}}}
\end{aligned}
$$

where, $\left(x_{\mathrm{A}}, y_{\mathrm{A}}\right)$ and $\left(x_{\mathrm{B}}, y_{\mathrm{B}}\right)$ are the coordinates of point $A$ and point $B$, respectively.

\section{CALCULATION ANALYSIS}

Based on the analysis with the mathematical model established above for the swing arm type DCG truck, the kinematics and force of the $20 \mathrm{~T}$ garbage truck are solved. The technical parameters of a 20 TDCG truck are shown in Table 1 [8].

The curve of the angle by which the lifting arm rotates is shown in Fig. (6). When the garbage container is unloaded, the lifting arm rotates from $0^{\circ}$ to $134^{\circ}$. That is, when the garbage container is unloaded, the lifting arm has to rotate by $134^{\circ}$. The movement trajectory of the center of gravity is shown in Fig. (7). It can be seen that the $X$-coordinate of the center of gravity of the garbage container changes from $1467 \mathrm{~mm}$ to $3655 \mathrm{~mm}$, while the $Y$-coordinate changes from $2562 \mathrm{~mm}$ to $1219 \mathrm{~mm}$. 
Table 1. Technical parameters of $20 \mathrm{~T}$ detachable container garbage truck.

\begin{tabular}{|c|c|}
\hline Item & Parameter \\
\hline \hline Tonnage & $20 \mathrm{~T}$ \\
\hline Rated loading weight $(\mathrm{kg})$ & 17750 \\
\hline Dumping angle $\left({ }^{\circ}\right)$ & $\geq 45$ \\
\hline Loading $/$ unloading time $(\mathrm{s})$ & $\leq 60$ \\
\hline $\begin{array}{c}\text { Size }(\mathrm{excluding} \text { the container): } \\
\text { Length } \times \text { width } \times \text { height }(\mathrm{mm})\end{array}$ & $9525 \times 2500 \times 3150$ \\
\hline Wheelbase $(\mathrm{mm})$ & $1850+3400+1350$ \\
\hline Front suspension $/$ rear suspension $(\mathrm{mm})$ & $1500 / 1425$ \\
\hline Angle of approach $/$ departure angle $\left({ }^{\circ}\right)$ & $30 / 15$ \\
\hline Height of hook center $(\mathrm{mm})$ & 1570 \\
\hline
\end{tabular}

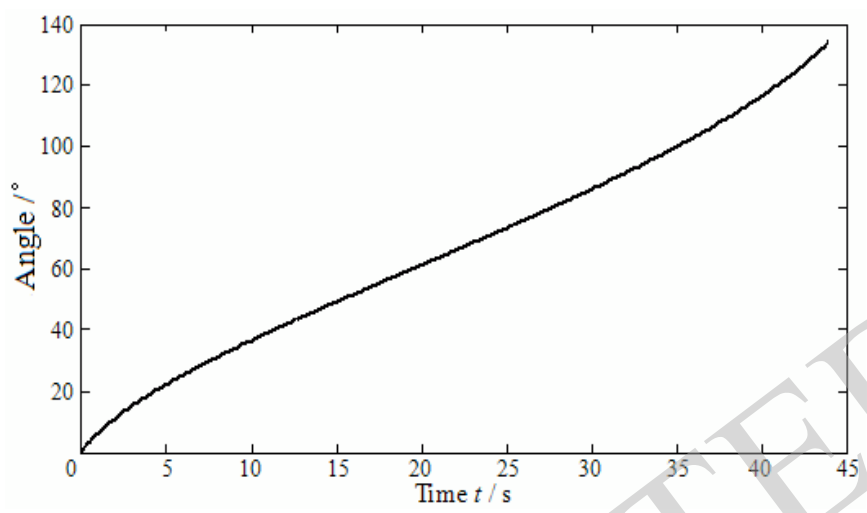

Fig. (6). Variation curve of the angle of lifting arm.

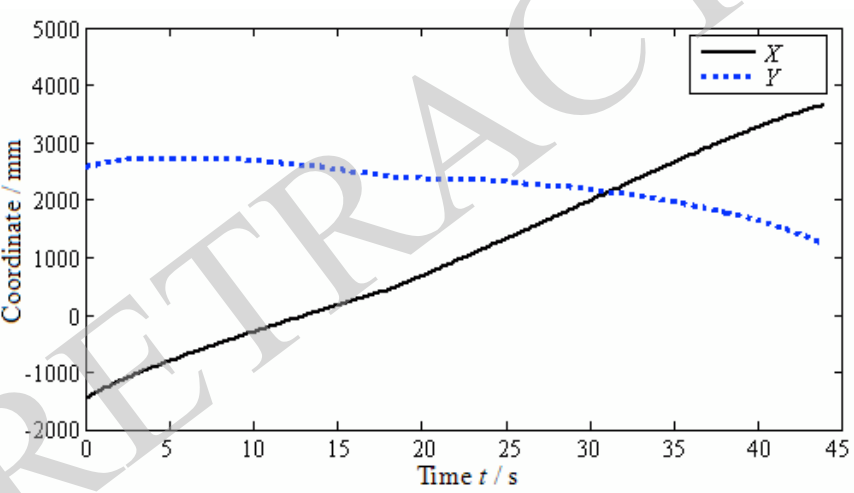

Fig. (7). Curve of the position of the center of gravity of container.

When the garbage container is unloaded, the force acting on the lifting cylinder in the first stage is shown in Fig. (8). When the lifting cylinder starts to move, the force acting on it is $-4.61 \times 10^{5} \mathrm{~N}$. When the lifting cylinder stretches, the force acting on it increases gradually. At $18.3 \mathrm{~s}$, the force reaches the maximum of $2.83 \times 10^{5} \mathrm{~N}$. The force acting on the lifting cylinder in the second stage is shown in Fig. (9). At the beginning of the second stage, the force on the lifting cylinder reduce sharply from $2.83 \times 10^{5} \mathrm{~N}$ by the end of the first stage ( $t=18.3 \mathrm{~s}$, the boundary between the first stage and the second stage) to $4.90 \times 10^{4} \mathrm{~N}$. After that, it increases gradually again. At $t=44 \mathrm{~s}$, the force acting on the lifting cylinder is the maximum of $7.33 \times 10^{5} \mathrm{~N}$ when the garbage container is dumped onto the ground.

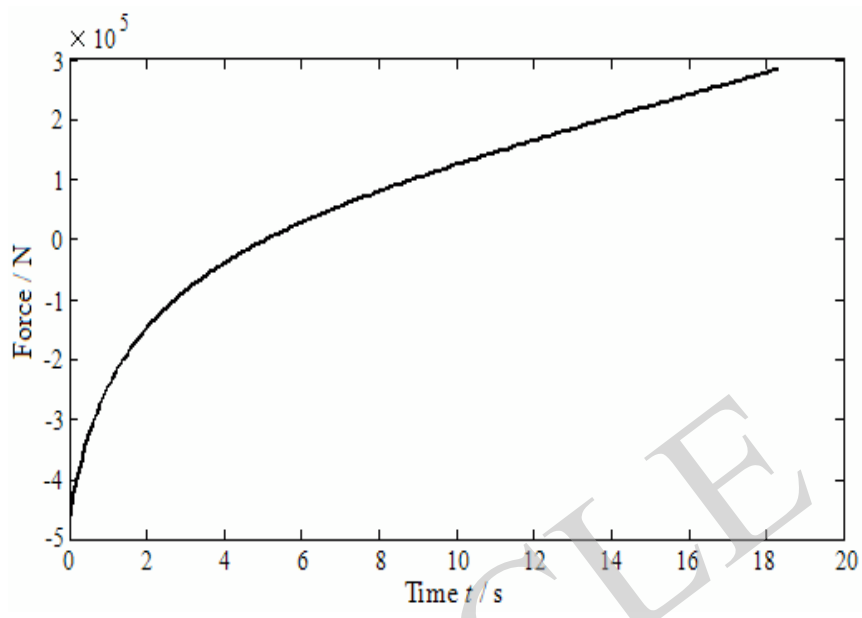

Fig. (8). Force curve of the lifting cylinder in the first stage.

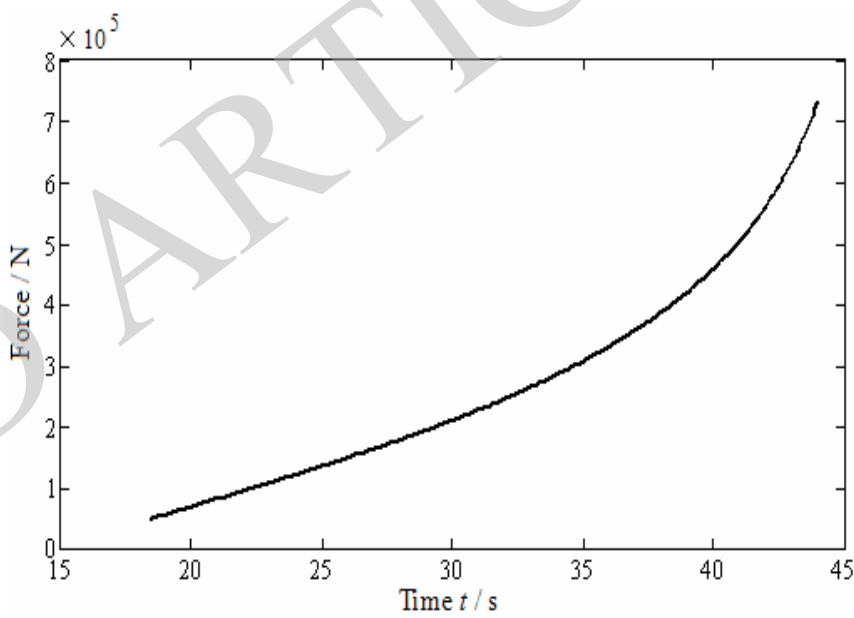

Fig. (9). Force curve of the lifting cylinder in the second stage.

When the garbage is dumped, the rotation angle of the flip frame and the garbage container is the dumping angle. The variation curve of the dumping angle is shown in Fig. (10). During garbage dumping, the dumping angle gradually increases from $0^{\circ}$ to the maximum of $45.5^{\circ}$. The movement trajectory of the center of gravity of container during dumping is shown in Fig. (11). The $X$-coordinate of the

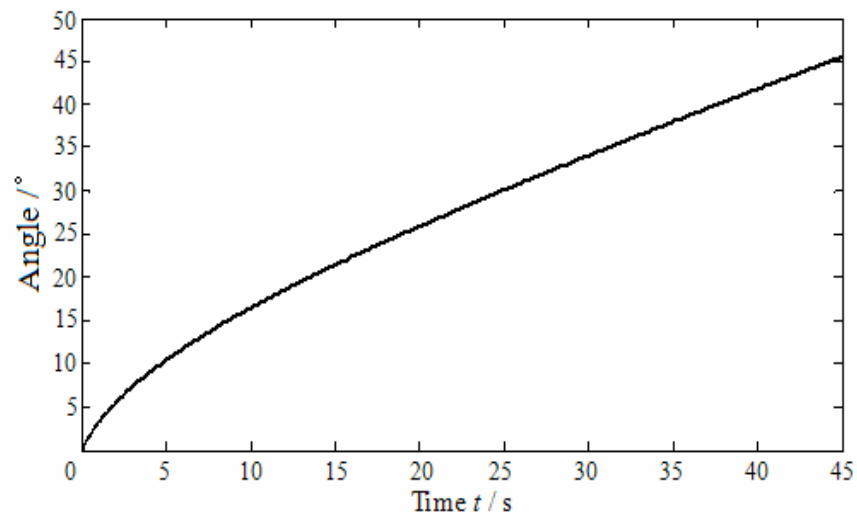

Fig. (10). Variation curve of dumping angle. 
center of gravity of container changes from $-1396 \mathrm{~mm}$ to $121 \mathrm{~mm}$, while the $Y$-coordinate changes from $2510 \mathrm{~mm}$ to $3147 \mathrm{~mm}$.

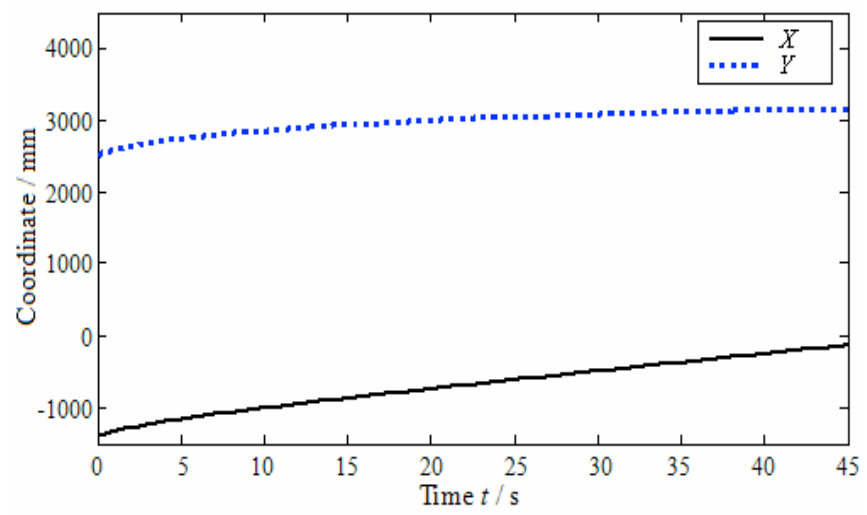

Fig. (11). Position curve of the center of gravity of garbage container.

During garbage dumping, the $Y$-coordinate of the highest point of the container ( $\mathrm{t}$ upper left point) is shown in Fig. (12). For various working conditions, the highest point is found during garbage dumping. When the lifting cylinder stretches completely, the upper left point of the garbage container has the maximum $Y$-coordinate of $6028 \mathrm{~mm}$. That is to say, the maximum height above the ground is $6028 \mathrm{~mm}$.

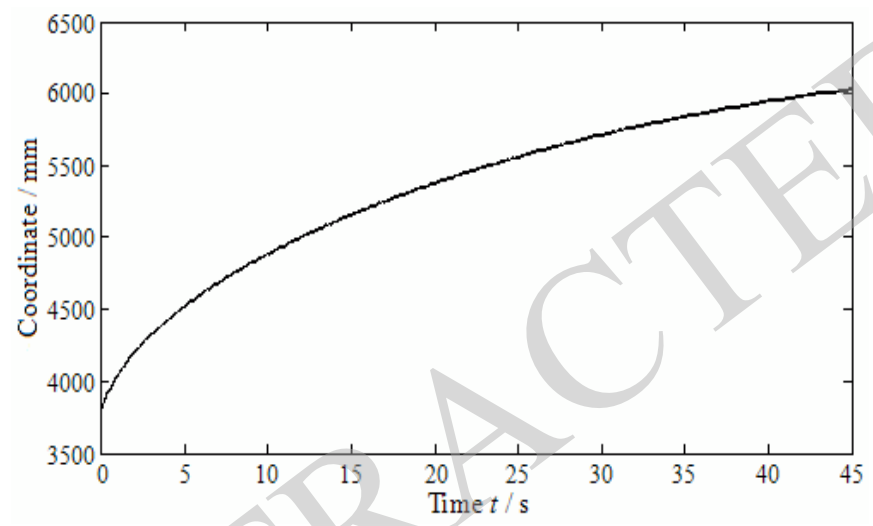

Fig. (12). $Y$-coordinate of the highest point of the garbage container.

The force acting on the lifting cylinder during garbage dumping is shown in Fig. (13). The maximum force of $5.49 \times 10^{5} \mathrm{~N}$ acting on the lifting cylinder occurs at the initial time during garbage dumping. The force gradually reduces to $8195 \mathrm{~N}$ as the lifting cylinder stretches.

\section{CONCLUSION}

(1) The kinematic and mechanical models of the lifting mechanism are analyzed during the two stages of unloading and under the working condition of garbage dumping.
(2) Using $20 \mathrm{~T}$ swing arm DCG truck as an example, the kinematics and force conditions of the lifting mechanism are calculated and analyzed.

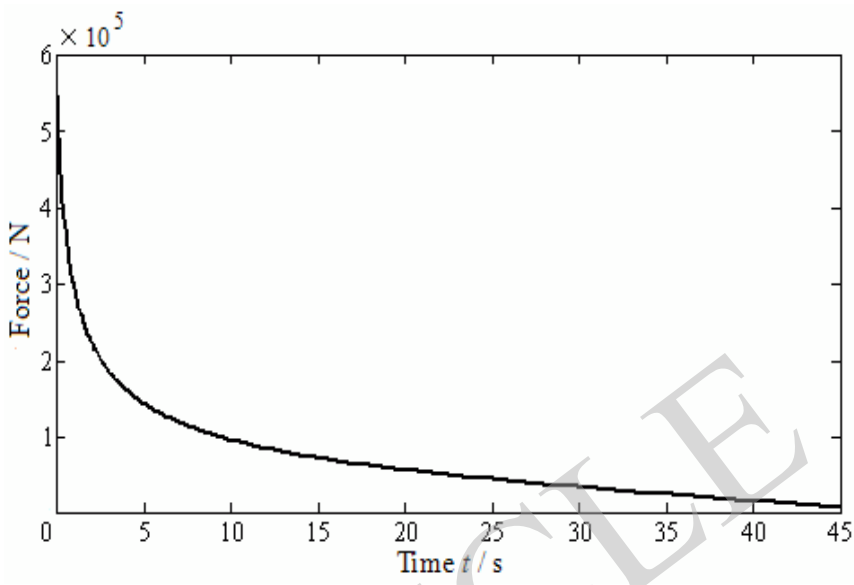

Fig. (13). Force curve of the lifting cylinder.

\section{CONFLICT OF INTEREST}

The authors confirm that this article content has no conflict of interest.

\section{ACKNOWLEDGEMENTS}

Project (2014QNB18) supported by the Fundamental Research Funds for the Central Universities of China.

\section{REFERENCES}

[1] Tao Sun, Qing Zhang and Ming Liu, "Design \& research of large height lifting mechanism for ultra-large structures", Advanced Materials Research, vol. 255-260, pp.634-638, 2011.

[2] R. Jiang, D. Liu, Z. Wang, and W. Fan, "Dynamic characteristics simulation for lifting mechanism of dump truck based on virtual prototype", Applied Mechanics and Materials, vol. 195-196, pp. 754-757, 2012.

[3] J. Hu, W. Ding, and H. Deng, "Dynamic modeling and analysis of lifting mechanism for forging manipulator", Applied Mechanics and Materials, vol. 278-280, pp. 633-640, 2013.

[4] S. Zhang, J. Zhang, and X. Li, "Optimal design of the front push rod assembly lifting mechanism on mining dump truck", $J$. Zhengzhou Univ. Eng. Sci. (China), vol. 34, no. 2, pp. 125-128, 2013.

[5] Z. Chen, Y. Wen, and Q. Liu, "Efficient global structure analysis of hook-lift device for detachable compartment refuse collection vehicle", Journal of Xuzhou Institute of Technology (Natural Sciences Edition), vol. 28, no. 4, pp. 66-70, 2013.

[6] X. Wang, Z. Huang, and H. Wang, "Research on characteristics of rotating hooklift", Machine Design and Research, vol. 19, no. 5, pp. 80-83, 2003.

[7] X. Wang, Z. Huang and H. Wang, "Optimization design on rotating hooklift", Journal of Tongji University, vol. 31, no. 9, pp. 10771081, 2003.

[8] L. Zhang. "Study on Pull Arm of the Detachable Garbage Truck", M.S. thesis, China University of Mining and Technology, Xuzhou, China, 2012. 